collected data to evaluate changes in practice. However, it is challenging to identify subtle changes in management such as better targeting of care without changing its frequency. In future, imaging investigations and changes in management should be identified more accurately by linking to the Diagnostic Imaging Dataset and prescribed medications.

\section{P85 EXCLUSION OF COMMUNITY-LED INITIATIVES BY PUBLICATION BIAS: EVIDENCE FROM A SYSTEMATIC SCOPING REVIEW OF COMMUNITY ENGAGEMENT IN THE UK}

AM Bagnall*, J South, J Trigwell, K Kinsella. Centre for Health Promotion Research, Leeds Beckett University, Leeds, UK

\subsection{6/jech-2017-SSMAbstracts. 186}

Background A recent systematic review found that initiatives with high levels of community engagement may produce more positive health outcomes than those with lower engagement. Systematic reviews in this area risk publication bias because (i) literature on community based health initiatives is widely dispersed and poorly indexed; and (ii) professionally-led (top down) interventions are more likely than community-led (bottom up) interventions to be formally evaluated and published.

An opportunity to examine the gap between research and practice arose in a systematic scoping review commissioned by the UK National Institute of Health and Care Excellence (NICE), of current practice in community engagement.

Methods We searched specialised research registers and websites; literature searches and citations from recent relevant systematic reviews; and direct calls for evidence via networks of community practitioners and groups. Records were screened independently by more than one reviewer, and included if published after 2000, relevant to the UK and evaluated or described community engagement in public health. Included records were coded for type, level and extent of community engagement, indicators of disadvantage, issues and outcomes.

Results 316 articles were included, 72\% were research or evaluation studies $(7 \%$ were randomised controlled trials; most were mixed methods or qualitative studies). $26 \%$ were found through website searches and the calls for evidence. The issues addressed most frequently were social capital or social cohesion (41\%) and community wellbeing (35\%). Indicators of health inequality observed most frequently were socioeconomic (39\%) and "other" (39\%), including people with disabilities; refugees and asylum seekers; mental health service users. Only 33 initiatives reported a high extent of community engagement; a comparatively high proportion were in the nonresearch literature $(20 \%$ of non-research articles, compared to $8 \%$ of research articles). This may indicate a gap between organisations which usually write and publish research articles, and organisations which fully involve community members, and/or may indicate challenges in the evaluation or publication process of high community engagement initiatives.

Conclusion Using conventional systematic review methods to examine community based approaches risks overlooking community-led "bottom up" initiatives, which may have the highest potential to reduce health inequalities. Reviewers should therefore make every effort to find reports of such initiatives, and consider broadening their definition of "evidence".

\section{P86 ARE PROCESS EVALUATION MEASURES RELATED TO INTERVENTION OUTCOMES IN THE PACE-UP PRIMARY CARE PEDOMETER-BASED WALKING TRIAL?}

${ }^{1} \mathrm{C}$ Furness ${ }^{*},{ }^{1} \mathrm{E}$ Howard, ${ }^{1} \mathrm{E}$ Limb, ${ }^{1} \mathrm{DG}$ Cook, ${ }^{2} \mathrm{~S}$ Kerry, ${ }^{1} \mathrm{C}$ Wahlich, ${ }^{3} \mathrm{C}$ Victor, ${ }^{4} \mathrm{U}$ Ekeland, ${ }^{5} \mathrm{~S}$ liffe, ${ }^{1} \mathrm{M}$ Ussher, ${ }^{1} \mathrm{M}$ Ussher, ${ }^{1} \mathrm{P}$ Whincup, ${ }^{3} \mathrm{~J}$ Fox-Rushby, ${ }^{1} \mathrm{~J}$ Ibison, ${ }^{1} \mathrm{~S}$ DeWilde, ${ }^{1} \mathrm{~T}$ Harris. ${ }^{1}$ Population Health Research Institute, St. George's University of London, London, UK; ${ }^{2}$ Pragmatic Clinical Trial Unit, Queen Mary's University of London, London, UK; ${ }^{3}$ Gerontology and Health Services Research Unit, Brunel University, London, UK; ${ }^{4}$ Department of Sports Medicine, Norwegian School of Sports Science, Oslo, Norway; ${ }^{5}$ Research Department of Primary Care and Population Health, University College London, London, UK

\subsection{6/jech-2017-SSMAbstracts. 187}

Background PACE-UP trial results demonstrated positive effects of a pedometer-based walking intervention on objective physical activity (PA) outcomes at 3 and 12 months in 45-75 year old primary care patients, in both postal and nurse-supported trial arms compared to controls. We explored associations between intervention implementation measures and change in PA outcomes.

Methods In accordance with the MRC guidance and framework (2014), the methods were selected through a key function model. Three quantitative aspects of the process evaluation relating directly to PA outcomes at 12 months were identified to assess intervention implementation: nurse session attendance (dose); PA diary completion (fidelity); and pedometer use (fidelity). These were considered as independent variables in the multi-level models estimating the effectiveness of the intervention on PA outcomes (changes in step-counts and time in moderate-to-vigorous PA (MVPA) levels in $\geq 10 \mathrm{~min}$ bouts).

Results Dose participants attending all 3 nurse sessions increased their step-count at 3 months by 961 steps more than those attending 0-2 sessions (95\% CI 401-1520, $\mathrm{p}=0.001)$. Minutes of MVPA were also significantly increased by $64(36,92)$ at 3 months and by $28(1,54)$ at 12 months. Fidelity: both postal and nurse groups showed strong positive associations of diary return on step-count and minutes of MVPA at 3 months compared with those who didn't return the diary: postal steps 1458 (854, 20161), nurse steps 873 (190, 1555), postal MVPA 64 (33, 94), nurse MVPA 47 (17, 75). These differences had decreased by 12 months, and only the postal group effects remained statistically significant: steps 1114 (538, 1689), MVPA 47 (17, 75). Regular pedometer use in the postal group was associated with higher step counts at 3 and 12 months: 1029 (383, 1675) and 606 (22, 1990) respectively. Regular pedometer use was not associated with PA outcomes in the nurse group.

Discussion Process evaluation measures showed significant associations with most PA outcomes at 3 and 12 months. These were stronger for the postal than the nurse group for diary and pedometer use. We cannot infer causality from these results, but the strong associations between nurse appointments, diary return, pedometer use and PA outcomes suggests that they were important factors in enabling the trial changes observed. We have shown the MRC framework to be an effective tool for process evaluation of intervention implementation. 